\title{
Quasi-omniscient Networks - Scenarios on Context Capturing and New Services through Wireless Sensor Networks
}

\author{
Rui Aguiar, Diogo Gomes \\ Universidade de Aveiro, Instituto de Telecomunicações, Portugal
}

\begin{abstract}
This paper addresses future scenarios for the telecommunications field, addressing the impact of three important research trends in computer networks: Context, Sensors and Wireless Networks. The proposed scenarios clearly highlight the possible synergies between the defined areas, and describe the role of Users and Network Operators in order to achieve the described goals. The potential danger of an over-encompassing network is identified, with a brief discussion on the challenges associated to the implementation of such a knowledgeaware communications network. The paper finalizes presenting a classification on the typical scenarios to be expected, and highlighting the associated challenges. It also presents a proposal on a scalable network infrastructure for Context processing.
\end{abstract}

Keywords: scenarios, context, wireless, sensor networks, network.

\section{Introduction}

The Information Society is characterized by a wealth of information. The existence of communication access (almost) everywhere, the pervasive access to Information Technology capabilities, the increased society reliance on computer-to-computer communications even for basic tasks (e.g. money transactions), all this has changed significantly the relationship between persons and their surrounding environment. Our citizens are now using regularly much larger amounts of information than what has ever happened in the past.

This information could nonetheless be considered a hindrance if humans could not contextualize such information and therefore filter only the relevant information to each individual. Computers on the other hand can be overwhelmed with information and through techniques such as Data Mining can extract a greater degree of relevant information more than a Human could ever could. But albeit all major advancements in recent years in Artificial Intelligence, computers are mostly considered dumb due to their poor capability to extract and network such information.

This "dumb" label is mostly due to the impressive human ability to contextualize information. Human ability to contextualize information comes mostly from its 5 senses (Sight, Taste, Touch, Hearing and Smell) - and these have not changed as time goes by. Sensing enables humans to interpret information according to the environment they are in and therefore make better use of the available information. Besides sensing their environment humans transform environment context into "useful" information they often share amongst themselves through communication (networking). 
In the last decades computers have been extended with networking capabilities that ultimately led to the Internet, but have always lagged in what concerns sensing. This changed in the last years, where we have witnessed great developments in the area of sensors. Besides their great capacity to process large amounts of information, computers have been fit with enhanced sensing capabilities compared to humans, and make use of new kinds of sensing not directly available to humans. Examples of such novel sensing concepts are Magnetoception (compass), Echolocation (sonar), Location (global and relative position), and Proximity. Information retrieved from such sensors can enable computers to have an augmented reality, and contextualize their outputs, which in turn is useful mostly if shared with humans who can take advantage of such reality. Making such realities widely available is therefore a key factor in determining the usefulness and importance of such extended realities. Due to the mobile nature of Humans, it is required that this information should be made available everywhere. It is in this scenario that Wireless Networks play an important role in distributing information among actors as they are a pervasive and widespread technology in our society.

In this framework, technology can act as a catalyst, especially when new sensing realities come into play. Since information is considered one of the main leverages to society change, by creating mechanisms that generate extra information and by ensuring its efficient distribution, society can be changed by technology. A very simple example is the impact that GPS technology had on our current life, with computers performing most navigation tasks in multiple transportation systems (air, sea).

The concept of ubiquitous computing [8] implicitly requires that computers are able to sense their environments and process information with minimum human intervention. The information retrieved by such sensors is then used to create context. Context which can trigger actions by computers, therefore coining the term "context-awareness" introduced by Schilit (1994) [1].

Obviously, one of the most interesting contexts to humans are humans themselves (proximity, mood, preferences). Technology can play an important role in this case as it allows humans to generate more context through networking: the information on how humans access new context information and how they act upon it, can be used to create (and broadcast) new (context) information. If this becomes a widespread action, then context can be exchanged in large amounts - and networks become easily involved in this process (for another simple example, the fleet location products now common in most countries). The network itself can track large context variations, and this in turn can be used to generate new context for the users.

This paper will address the possible impact of Context in society through several use-cases scenarios. In particular the merger of Wireless Sensor Networks with Telecommunication Networks, providing a rich context environment, is considered in this paper. In the next section we address the impact of context on telecom operations. In section III, we describe five major use-case groups, addressing services built on top of the availability of large amounts of Context information (retrieved through, but not only, wireless sensor networks), and present a Framework for classification of these scenarios. In section IV we address how network operators could incorporate Context Information into their existing and future network architectures. Finally, in Section V, we draw some conclusions on the possible realization of these scenarios, and on the impact of such a converged Context-aware Telecommunications Network. 


\section{Telecommunications and Context Usage}

The notion of context is often controversially defined, but loosely speaking, it can be considered as all background information that enhances the effectiveness of a given task, (e.g. metadata, logical business models, links to a function or activity, secondary data). Of course, in this definition, the controversial aspect is the notion of "background" - different purposes might see the same information with different relevance.

A more general view just assumes that all tasks act on a context - all information is considered context to the task. Taking this more general view simplifies analysis, and the definition of "context" becomes moot. Humans consume context, coming from various sources; the networks do as well.

The relationships of context and telecommunication networks are then complex. The networks can act:

i) as conveyors of context information between users, between users and their environment, and between both users and the environment and network service providers.

ii) as consumers of context, receiving information on what environment users are in, and adapting itself to this environment (e.g. users movement).

iii) as providers of context, providing information to all the consumers above mentioned about both network and user status (e.g. the network can provide location information for users, as well as the status of end-to-end connections)

Currently, telecommunications networks are already acting on all these fronts. With increasing machine-to-machine communications, telecom operators are routinely and transparently supporting applications that provide information such as location or proximity to users and systems. In fact, most often the content of that information is opaque to the communication network, which simply transfers bits.

The network as a context consumer is something that has been embedded in network planning from its inception, but it is not usually addressed. The environment (economic, density, profile) where a network deployment is to be made has always been the prime factor on network planning. However, this knowledge has been always "assumed" as static, or so slowly variable that no immediate impact on the network was to be felt.

Networks have also acted as context providers for long - namely for internal control mechanisms. The current load in cells, e.g., has been used in admission control regularly, and this context has been used for optimally provide certain quality-aware services.

It is clear today that users, networks, and service providers (both regular service providers, or those specialized in context-provision) will provide and consume context information. With the introduction of a multitude of sensors, and of sensor networks providing a multiplicity of context, networks will have to adapt themselves. The widespread introduction of wireless sensor networks, and of large and diversified sources and consumers of context, will allow for finer granularity in service provisioning, thus requiring more flexible and faster control loops in service provisions - including for current simpler transport services.

Basic network aspects, such as Mobility, QoS, or Security, can/will be affected by context information. Context, such as movement (position, speed) can be key to new mobility solutions, both for new services ('plane is late') and optimizations of current ones ('cell coverage'). In a similar way, QoS and Security features on the network react to context - the presence in a cell of specific users can bound the constrains for providing pervasive QoS, and 
the mechanisms for opportunistic security schemes, with variable security levels. Note that for the current integration trends in communications networks, these features need to be deployed not only in cellular networks $(3 \mathrm{G})$, but across the whole range of (wireless and wired) networks that are part of that operator offer - vide the convergence on the customer databases between cellular and hotspot subscribers, with cellular operators offering similar customer access in their hotspot division.

These changes to control mechanisms are nevertheless slow in entering the telecommunication network. The presence of new traffic flows is a trend much more demanding. The whole cellular network is now seamless integrated as a component on some sensor networks - although very specialized. The most common example are fleet location services, which have a sensor (GPS) inside the fleet vehicles, and use SMS or GPRS communications to perform location update in a server. In this case, the cellular network became the communication links for the sensor network, definitely an unexpected result of the increased need for more context information. More and more, the pervasive coverage of cellular networks, and the low bandwidth requirements of most sensor networks, are making this trend a reality, with cellular networks acting as transport mechanism for remote sensing, with aggregation units centralizing the distilled information of sensors (or of coupled sensor networks).

Considering these already existing trends, the following section will present some extrapolated scenarios on features and services achievable on strongly coupled sensor and communication networks.

\section{Context-aware Scenarios for Telecommunications}

When discussing novel scenarios to be exploited by the merger of wireless sensor networks and telecommunications networks we are addressing Next Generation Networks scenarios. The visions here assume beyond-3G networks, mostly in urban environments with many sources of context everywhere. These beyond-3G cells will probably be very small (depends on the technology, e.g., a cell of IEEE802.11 may have a few tens of meters while a single DVB base station may cover a whole country). Many terminals will have multiple interfaces, and will be able to scan its surroundings though multiple technologies. Multiple network and service providers will exist, and the user may be simultaneously using these different providers - and often even using different terminals (or terminal interfaces) simultaneously. Terminals will be able to explore information on RFID devices (e.g.) or coming from Wireless Sensor Network providers. In this world, several scenario types can be envisaged.

\section{A. Context Aware Services}

Context aware services are by no means an innovative conceptive. Commercial examples abound that make use of location context based on geo referencing, be it GPS or network coverage. Such services are very intuitive and appreciated by end users and prove the usefulness of Context aware services, but nonetheless fallback on the possible expectations by users and network operators alike. The users often expect a "god-alike network", which is able to provide the needed information without any hurdle. With the expected deployment of sensors in an increased number of network enabled consumer devices, it is expected that network context can allow networks to reach a quasi omniscient level where the network can provide for all of its users needs in an efficient way.

One of the most important aspects in today's networks is the perceived Quality of Experience. Quality of Experience considers not only the Quality of Service the user gets in 
terms of network performance but also how is this quality experienced by the end-user. Does he acknowledge the quality received? Does his equipment fully support the service delivered? Just considering a simple multimedia stream, the impact of environment factors that may affect the user perception of the service quality is quite large: video and audio capabilities of the device, existing sound and lighting conditions, current user activities (moving? driving?), etc... These and other questions can only be handled if context is part of the equation:

\section{Context (Service) $-\operatorname{Adapt}_{N E T}[$ Context (NetworkConditions) $]-\operatorname{Adapt}_{U S E R}[\operatorname{Context}($ User $)]=\mathrm{QoE}$}

Service is in this case considered the "perfect" reference point which is negatively impacted by the Network Conditions (defined by QoS) and by the User Environment (lighting, noise and terminal definitions). In this equation, these context conditions lead to compensation actions from the network operator, or from the user terminal. Context acts as the control variable that can be used by the network to maintain QoE by compensating on the Network Conditions or the definitions of the Service, or that can be used by the terminal software to adapt to the user conditions.

Common are our modern life scenarios where we are in a noisy environment where audio services could be delivered with less quality without any perception by the user of such drop, but with immediate impact on the network due to a more efficient use of resources. The same could be said of a video that due to the amount of light in the user environment could be delivered using different codecs to assure the best perception of image under such harsh environment, or that should be stopped and moved to audio only because the user is driving [9]. This scenario obviously relates to context aware user-services that adapt the offerings to the user based on the user status (a Service Provider can make use of its service bucket to better target its end user with the appropriate content). Ultimately this context information could be used in context aware routing of packets where packets are no longer treated according to network parameters but according to human relevant context (Context aware QoS, where differentiation is done on relevance of packet content and not on packet headers ${ }^{1}$ ).

Context can also influence Multicast Service delivery, impacting multicast tree construction. The multicast trees could be built according to the service and available transport technologies, introducing added intelligence in the tree construction algorithms. In such a scenario, group unidirectional services (e.g. TV broadcasting) would make use of unidirectional technologies (e.g. DVB) while services with user interaction would build multicast trees based on bidirectional technologies. This feature could leverage the use of Broadcast technologies that are sometimes not chosen due to the difficulties involved in integrating them with interactive services.

Furthermore, emergency situations could trigger specific network profiles, which could be used as alerts besides the normal emergency measures. A Fire department would be able to reach fireman on license even under heavy network congestion. Note that albeit some provisions exist on legal requirements for telecom operators to support catastrophe situations, these actually are only foreseen for war situations in which a human must intervene to change the network. With context-aware services embedded in the network, these priorities could be

\footnotetext{
${ }^{1}$ Naturally, efficient methods for deep-packet inspection/packet marking would be required for such network features.
} 
inherent on the network behavior, and added QoE could be provided to critically needed users.

\section{B. Intelligent network control}

Added information can help networks to perform better. This will ease basic network tasks such as mobility, QoS and security, as mentioned in the previous section, which can benefit of knowledge about the user activities. These are already existing trends that can only be exacerbated with increased coupling with sensor networks. Also the scenarios depicted previously in section III.A, on Context-aware Services, included themselves a certain degree of network control change.

However network control can benefit much more from added context information, and knowledge on users. An example of how far this trend can lead us to is the field of network planning. Network planning aims at ensuring that a network or service meets the needs of the subscriber and operator. This task is increasingly difficult as needs are increasingly diverse, and user's mobility increases. A miscalculation could mean under provisioning or overprovision of the network, with its direct financial impact on the operator. Depending on the context of the region the network planner must forecast the networks needs, in a task that is neither complete nor reliable.

This human context on which the network is deployed plays a key factor, and places such as large sport stadiums pose a challenging task. During games the network will for sure be under provisioned (with loss in possible revenues for the operator and frustration to the user), but on the remaining days the network will be over provisioned. With dynamic cell coverage, scenarios can be thought where this coverage is varied (either towards the stadium or its proximity). Information about events occurring in the stadium collected through not only sensors in the stadium but also by context retrieved beforehand from $3^{\text {rd }}$ party services (such as ticket selling points) could inform the network operations division of the need to increase/decrease capacity by rearranging the network (antenna and cell control). In a more futuristic scenario we could consider meshed networks, with base stations interconnected in a such a way that the total capacity would depend on the context of the network users (by day with increased coverage closest to the work place, by night closest to entertainment and residential areas).

\section{Environment-aware Terminal}

As mentioned in the introduction sensors and context are key properties in the concepts of ubiquitous and pervasive computing. Nonetheless applications have limited themselves to small environments and minimal networking of context between environments. The most common scenario, several times depicted in literature, is the intelligent home/office where sensors control ambience variables such as lighting and temperature based on sensors distributed around the building. Integration of communication devices is also referenced [10], and involves the possibility to make use of the house/office to communicate presence and/or location.

With the integration of communication networks in pervasive computing [7], more ambitions scenarios can be considered, and still retain this "device-centric" approach. The network can act, e.g. as a context provider, feeding the information on the users to its environment, leading to network(ed) context.

Besides basic sensor based context, this networked context can be used to create new services and enhance inter-human communication. One very simple scenario is one such that 
a communal TV on a Pub presents a program that is function of the personal profiles of the users present in the room.

Note that this profile information may be provided through its network operator, or through direct communication between the users and their devices. This scenario reflects a sort of automatic "majority vote" for specific groups of users. In these environment-aware terminal scenarios, context comes from both the user and its environment and can be communicated to other users and environments.

The basic "terminal-centric" application cases are of little relevance for our merged communications and sensing scenarios. However, as a use-case example, we can consider a world in which Context awareness can lead to networks that track the user around in its home, car, office and between places without carrying around a cumbersome terminal (this scenario resembles a popular "Beam me up, Scotty" Sci-Fi. scene). The terminal becomes yet another delivery mechanism for information coming from the network, and according to its environment, it can be "selected" as the "user device" -naturally informing the network of this (new) role.

\section{Terminal controlled by the network}

A different vision on the user-network interaction is to actually consider the terminal optimally configured by the network. In general, all modes of existing control of the terminal by the network would benefit from added information, leading to networks more efficient and improved terminal performance.

This already happens in terms of power control, e.g., in cellular networks. Modern life is filled with small devices with wireless communication capabilities that are very expensive in terms of power consumption. This well known aspect is in general matched by the power consumption of networks that require 24/24 availability. Making our networks and terminals more power efficient is therefore not only a desire but a requirement for future network architectures - and furthermore power efficiency improves transmission behaviour.

The use of wireless sensor networks (which are extremely power-efficient) can lead to efficient algorithms for power consumption control based on context. A first scenario would be futuristic terminals and networks where a close range technology could be used to sense terminals and on demand turn on the radio capabilities of the terminal [6] and change base station power control for the area. In this scenario zones or periods of time could be in a blackout mode where almost no energy is spent and turn on only when any communication is required to happen (by sensing the user presence or according to the service context). Another use-case could include context aware transmission modes. A non real-time service data could be cached in the Base Station and provided to the terminal on burst modes therefore efficiently using all the available bandwidth and limiting the amount of time the terminal interface requires to be switched on. Another pervasive use-case could consider a theater where the network shuts itself down during performance time except for emergency officials (doctors, firefighters, etc).

From another viewpoint, network initiated handovers [6] could be performed more accurately as the network would be in possession of more context information.

\section{E. Pervasive Sensing Environments}

Coupling sensor networks to communications networks can bring added dimensions to communications, expanding the current notions of virtual meetings. 
Multimedia Conferencing is one of today's most important collaboration tools as it enables participants to discuss and exchange ideas under a common context created by the shared distribution of audio and/or video. On a tighter analysis we can say that we have a virtual reality that is sensed by cameras and microphones, which create a meeting point context. Compared to the real life situation of a conference, this virtual reality is considered a diminished reality as it lacks full information (lack of context) on the status of the participants. Perception of partner's interest and attention rates are two important context elements that today's multimedia conferencing systems are unable to deliver.

In order to deliver an augmented reality of a conference, new sensors are needed to collect participant's information as well as networking mechanisms that might potentate communication such as temperature monitoring (comfort is an important element to get/maintain attention), dynamic parallel audio groups (enabling cross talking without interferences) that can be monitored for concentration levels of participants, as well as intelligent contextual push-to-talk mechanisms that support the conference chair's task.

Another example is the case of virtual worlds where people could actually see each other as they require, and not need to be always in "director's mode", supporting side conversations that could be kept private and concealed in order not to disturb the speaker but enabling quick exchange of ideas. A sensor network would provide information on the mood of the participants, track movement and activity of the interlocutors therefore providing context to the remaining participants.

In these examples, the existence of powerful context information coupled to communication networks would expand manifold the experience of distance communication. Note that this is not the case of adapting existing multimedia applications to the sensed environment, but to effectively incorporate new communication channels, transferring as much as possible all user's environment across distance.

\section{F. Framework for scenario classification}

These scenarios reflect some of the potentialities of merging telecommunications with wireless sensor networks. The scenarios have been classified along the five categories above. This categorization reflects types of interactions between which entities provide context, and which entities react to this context. Figure 1 depicts the relationships identified and structured along lines moving from Terminal+Sensor Networks to (Communication) Networks and Service Providers.

- In the Intelligent Network scenarios, the network is the element that is actually responding to the context changes, and retrieves most of this context from itself and from other Service Providers. This is a network-centric action, and the user is completely unaware of the actions.

- The Controlled Terminal visions are scenarios where the terminal itself is being controlled by the network, according to the global context, extracted mostly from the terminal and near sensor networks. Both network and terminal do act on these scenarios, which are terminal-centric, and may impact slightly user perception of the communication process.

- Context-aware Services are scenarios where the services being provided to the user are actually reacting to context, changes on overall environment. This is mostly an action on the provider's side, albeit this may also impact on the user device. These are scenarios that are network-centric, but that improve user perception. 
- A user-centric vision of this merger of sensor and communication networks is presented on the Environment-aware Terminal. Here we mostly have actions on the terminal side (including terminal movement), and most of the context will come from the terminal and its surrounding networks. Nevertheless, some actions from the Network side may be required.

- Finally, the Sensing environment presents the intertwined scenarios - here all elements of the communication process are used to extract context and all of them may be affected by this context: the communication network, the sensor networks, the devices and the services being provided.

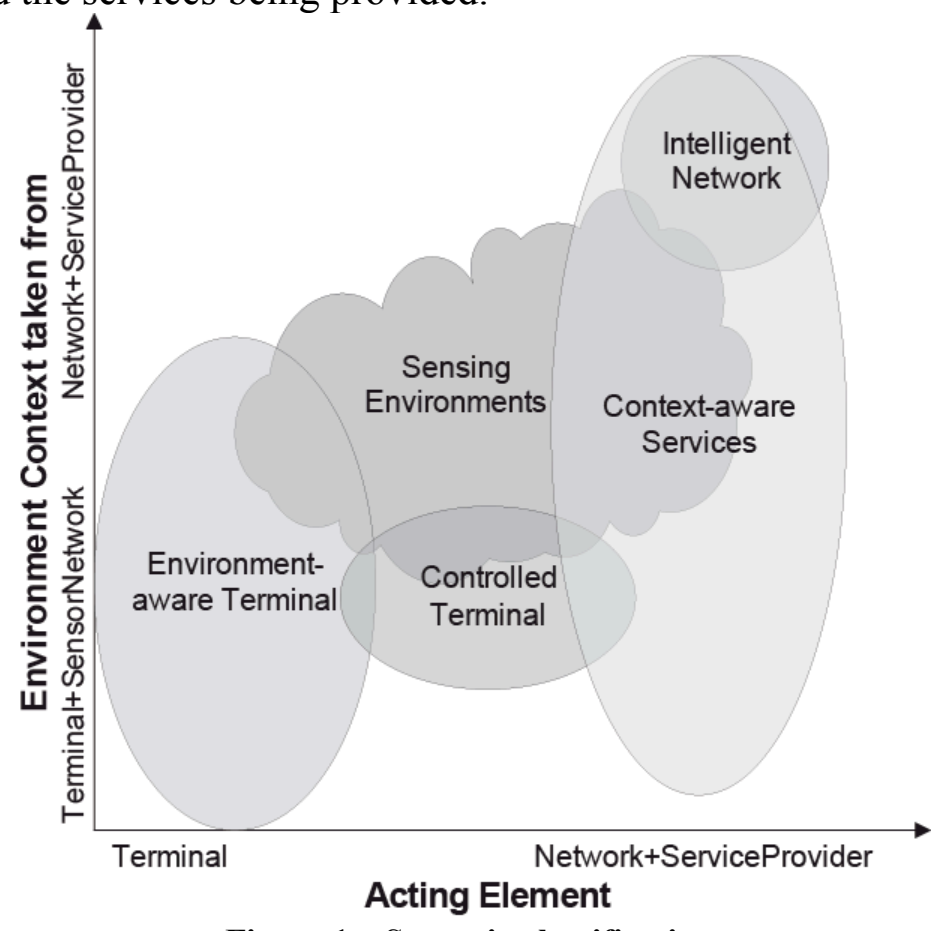

Figure 1 - Scenario classification.

It is readily seen that for these scenarios imply closely coupled networks, but with flexibility to adapt to different types of requirements.

\section{Scalable network infrastructure for Context Processing}

The overall system implicit on the scenarios above contains in a large measure intelligent systems features. The service(s) provided has to be adapted (in some sense) to the existing context, has to present different outcomes according to the conditions that are triggering the service. Note that this differs from traditional service inputs by two facts: i) the basic service functionality does not depend on the complexity of the intelligence modulating the service outputs; ii) and there is an (potentially distributed) infrastructure outside the service provision core, which provides a wealth of information and refines it to optimize the service outcome in fact this optimization process may be dynamic, and totally independent from the initial trigger that originally led to the service invocation.

The service provision relies thus on an intelligent reasoning system coupled with a data acquisition system, in fact including pervasiveness features in the network service 
infrastructure [7]. The telecom infrastructure has to inherently include sensor aspects in its internal service operation.

From a knowledge processing point of view, the system can be represented by Figure 2 . We have sensors, which perform data collection, which is then aggregated and preprocessed. All this information is then available in different units. The network telecommunication infrastructure then collects this information along two different lines. The Context and Costumer Management contains databases with (processed) context information, including context models, profiles and context data itself. Note that the management of the context itself can be a problem, requiring specific platforms for assuring database consistency (e.g. see [2])

Furthermore, customer information (user profile, his current attributes) is stored in its own database. Both sets of information are processed, in order to both infer situation and context, using reasoning. Naturally this may be a complex task - in fact will be as complex as the desired context-aware intelligence. In some cases, it may be adequate for doing this reasoning, and to unblock/refine some rule execution, that the reasoning accesses (pulls) specific information from the Sensor Aggregation units.

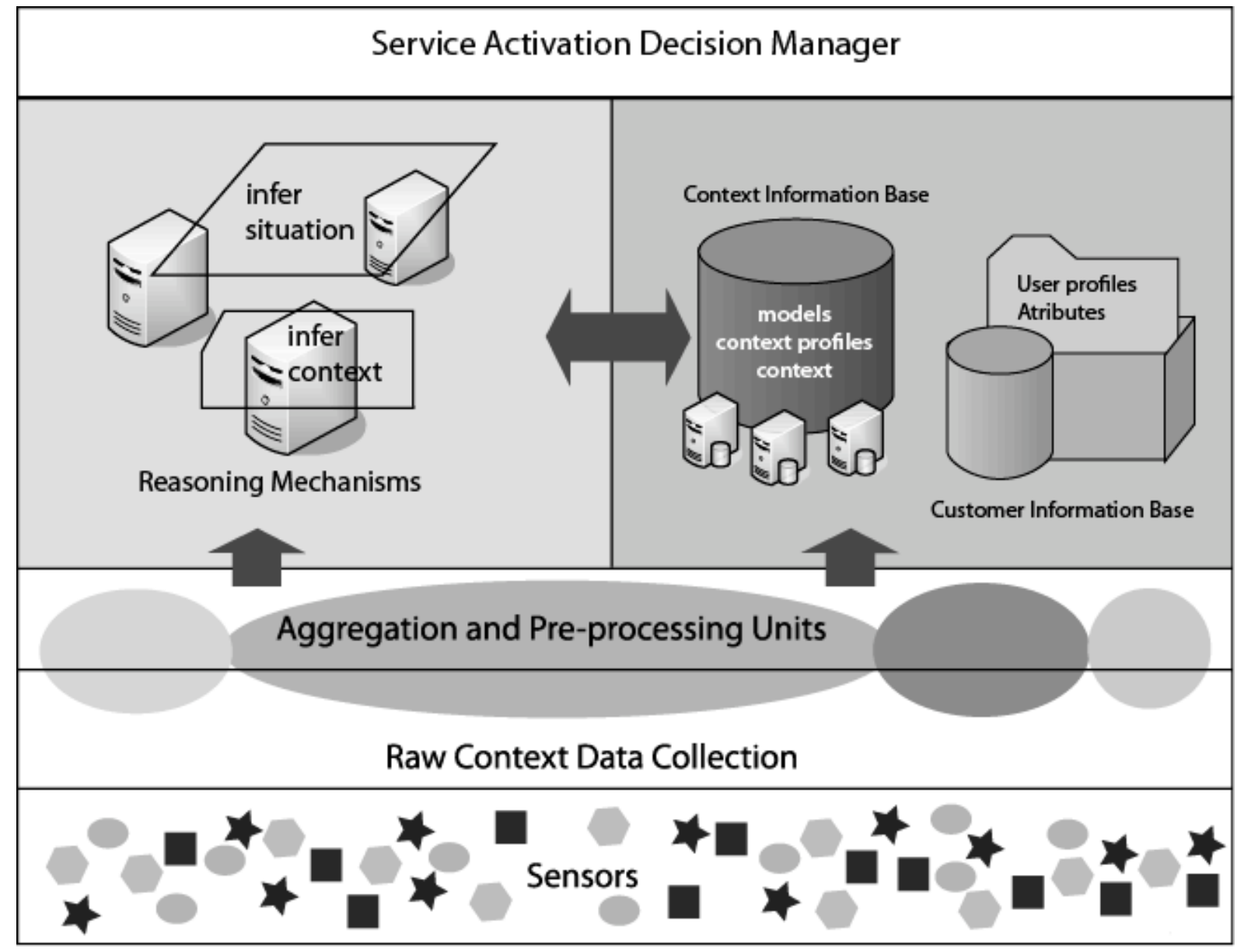

Figure 2- Information Processing View

The Service Activation Decision Manager then takes all these outcomes (the database information, the reasoning over this) in order to modulate the service response. Note that this manager can be embedded in the Service infrastructure, or may be itself a Service upon 
which other services are registered, and which provides configured triggers to the telecom services (see Figure 3). In fact, in general, both situations will exist.
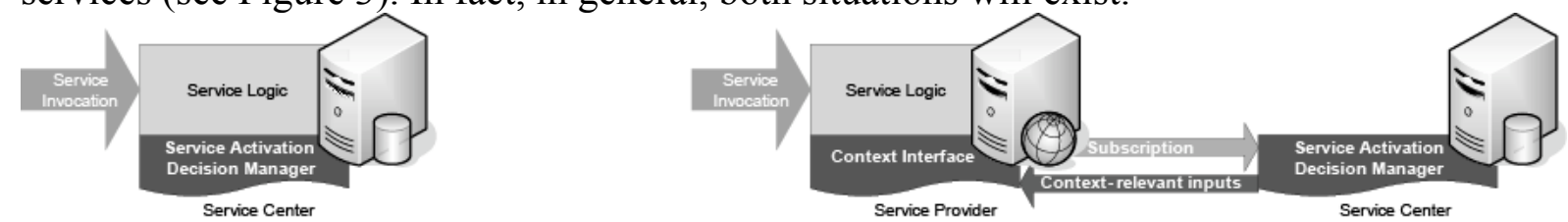

Figure 3 - Service provision and Context Processing: embedded service logic (left) and subscribed external support (right)

The previous discussion was based on knowledge processing models. The telecommunication infrastructure was not apparent in this process, neither the communication flows. Figure 4 presents a telecommunication representation of the information flows associated with this concept.

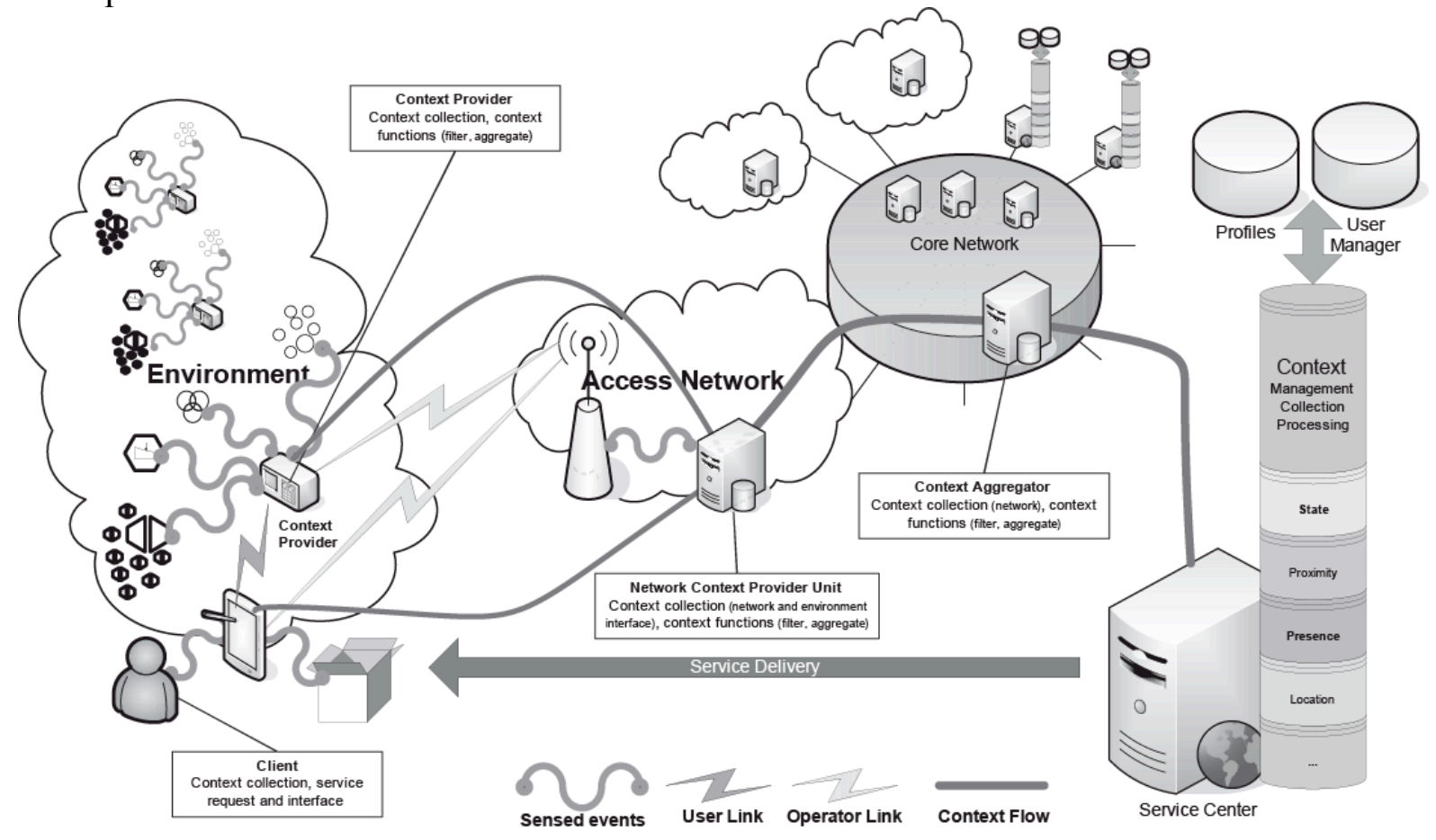

Figure 4- Context-aware Telecommunication Infrastructure

The Telecom Service Center (where all the central context processing resides) retrieves data from traditional profile and user management databases. Context arriving to this center passes through a large set of intermediate processing entities, required for scalability reasons. At the core network, we have a context aggregator that performs final coarse filtering on the data to pass to Service Center. This Context Aggregator is fed by multiple Network Context Provider Units. These units are associated to access networks of manageable dimensions, and are the first level network units for context acquisition and processing. Context can be stored in these units centrally or in a distributed manner (e.g. [4]). These units retrieve information from multiple sources: the network itself; the user client application; and from external sensing units (or context providers). Note that in this discussion, multiple Service Centers and Context Aggregators may exist. Different services may be processed by different Service Centers, as well as different context sources may be aggregated by different Context Aggregator Units. 
Furthermore, multiple sensor sources may exist. The user itself may provide relevant data (e.g. "mood"), or its communication device may either acquire data from nearby objects (e.g. by RFID), or from internal sensors (e.g. GPS location). Note that this device may also be connected to, and retrieve data from, context provision units [5]. These units (e.g. the gateway element in a sensor network) can also be a source of context to be used by the telecommunication infrastructure. In this architecture, both terminal-supported and networksupported [3] approaches to context acquisition and management are considered, and can coexist.

Note that this infrastructure, where higher level units subscribe from information provided by lower levels units, allows for a scalable deployment of personalized, context-aware, service deployment. Multiple entities are distributed at different levels, and in fact even the entities themselves may be deployed in a distributed manner (e.g. [4]).

\section{Conclusions}

This paper addresses the increasing requirements for an almost omniscient network - the user is increasingly expecting its environment to adapt to himself, proving emergency support, aid and advice in multiple situations. This trend, one that is being further challenged by increasing mobility of the users, seems to be one that can be realized by evolving current technologies.

Telecom operators can set up a scalable infrastructure which is able to benefit from independent deployments of wireless sensor networks, and interact with user devices. This infrastructure can actually exploit context in general terms, provided from multiple sources, from the network itself to the user device. The paper highlighted several scenarios which would benefit from this integration of context in telecommunication networks - and these benefits would be felt both by the network and service providers as well as by the end-users. These scenarios covered a large spectrum of usage and of involved entities. Next Generation Networks can exploit a large amount of context information, but managing the amount of data associated to an universal context-aware environment is a daunting task, most probably only possible to be addressed by exploiting existing expertise in managing large communication systems. Note that future pervasive services, context-aware, will only be realizable for the general user if practical management is possible.

Wireless Networks are nowadays Omnipresent, by integrating the context awareness as proposed in this paper they can become quasi-omniscient, thus coming closer to a all knowing entity. In general, this "omniscient network" seems to be realizable as an evolution of current infrastructures, but some concerns may exist regarding the degree of reactiveness of such a system - in particular regarding how much information is derived about a given user. This privacy issue is increasingly important, and is tackled in another paper in this issue named "Virtual Identity Framework for Telecom Infrastructures".

\section{Acknowledgements}

This work was sponsored partially by FCT grant BD/16244/2004, and was triggered by discussions inside the EU projects Daidalos and C-Cast. Several aspects of this paper (some of the scenarios) are being actively pursued and deployed inside these projects. Thanks are due to multiple partners by their opinions and discussions. 


\section{References}

1. B. Schilit, N. Adams, and R. Want. "Context-aware computing applications". IEEE Workshop on Mobile Computing Systems and Applications (WMCSA'94), Santa Cruz, CA, US: 89-101, 1994.

2. Inoue, M.; Hasegawa, M.; Morikawa, H., "Decentralized ubiquitous networking server for context-aware seamless services", IEEE 61st Vehicular Technology Conference, 2005. VTC 2005-Spring. 2005 Volume 5, Page(s):2805 - 2809 Vol. 5, 30 May-1 June 2005.

3. Loke, S.W.; "Context-aware artifacts: two development approaches", IEEE Pervasive Computing, Volume 5, Issue 2, Page(s):48 - 53, April-June 2006.

4. Ocampo, R.; Cheng, L.; Jean, K.; Galis, A.; Prieto, A.G.; "Towards a Context Monitoring System for Ambient Networks", First International Conference on Communications and Networking in China, 2006, ChinaCom '06, Page(s):1-3, 25-27 Oct. 2006

5. Hofer, T.; Schwinger, W.; Pichler, M.; Leonhartsberger, G.; Altmann, J.;

Retschitzegger, W. "Context-awareness on mobile devices - the hydrogen approach", Proceedings of the 36th Annual Hawaii International Conference on System Sciences, Page(s):10p, 6-9 Jan 2003

6. T. Melia, A. de la Oliva, I. Soto, P. Serrano, and R. Aguiar, "Network Controlled Handovers: challenges and possibilities", Wireless Personal Communications, DOI 10.1007/s11277-007-9256-5, Springer (in print).

7. Rui L. Aguiar, Amardeo Sarma, Dennis Bijwaard, Loris Marchetti, Piotr Pacyna, Riccardo Pascotto, "Pervasiveness in a competitive multi-operator environment: the Daidalos project", IEEE Communications Magazine, Large Projects section, vol 45 n.10, pp 22-26, Oct 2007.

8. Mark Weiser, "Some computer science issues in ubiquitous computing", ACM SIGMOBILE Mobile Computing and Communications Review archive, Volume 3 , Issue 3, Page: 12, July 1999.

9. Michael Angermann, Sarah Mcburney, Christoph Kuhmuench, Fiona Mahon, Jelena Mitic, Patrick Robertson, John Whitmore, "Integrating and Demonstrating Pervasiveness in a Scenario Driven Approach", eChallenges e2006 Barcelona, Spain October 06.

10. M. Friedewalda, O. Da Costab, Y. Punieb, P. Alahuhtac and S. Heinonend, "Perspectives of ambient intelligence in the home environment", Telematics and Informatics, Volume 22, Issue 3, Pages 221-238, August 2005

\section{Curriculum Vitae}

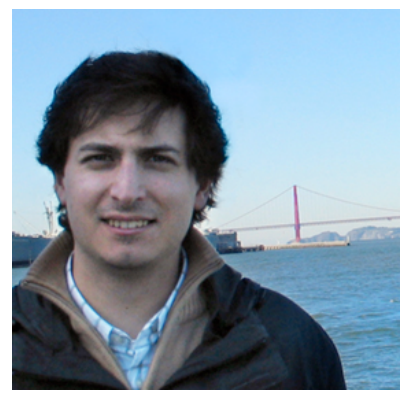

Diogo Gomes graduated in Computers and Telematics Engineering from the University of Aveiro in 2003 with first class honors, and has since been working towards a $\mathrm{PhD}$ degree in "QoS signaling in broadcast enabled technologies») at the same university. He has been invited lecturer at the Unviersity since 2007. Since his graduation year, he has participated in several IST Projects such as IST-MobyDick, IST-Daidalos and IST-Akogrimo where he handled QoS and mobility related issues as well as prototype 
implementations. Recently he became involved in the project IST-C-MOBILE, where he holds leadership responsibilities on the deployment of a proof of concept platform for the project. His research interests include Multicast, Service Platforms and QoS.

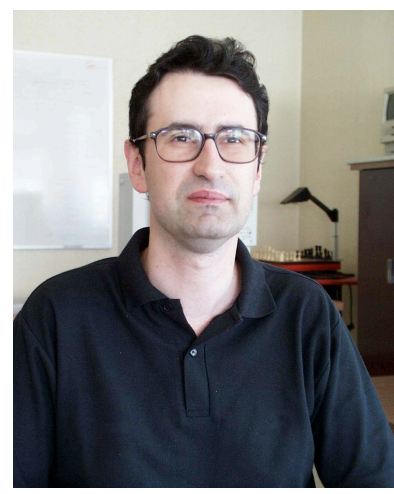

Rui L. Aguiar received a Ph.D. degree in electrical engineering in 2001 from the University of Aveiro, Portugal. He is currently a professor at the University of Aveiro, and a Guest Professor at Carnegie Mellon Cylab. He is leading a research team at the Institute of Telecommunications, Aveiro, on next-generation network architectures and protocols. His current research interests are centered on the implementation of advanced wireless networks, systems, and circuits, with special emphasis on QoS and mobility aspects. He has more than 200 published papers in those areas. He has taken chairing responsibilities in several conferences, such as ICNS'05, ICT'06 and ISCC'07. 